\title{
Case Report: Concurrent Sustentaculum Tali and Lateral Talar Body Fracture in a 19 Year Old Patient
}

\section{loannis M Stavrakakis $^{1^{*}}$ (D), George E Magarakis ${ }^{2}$, Zacharias Christoforakis ${ }^{1}$ and loannis Daskalakis ${ }^{3}$}

${ }^{1}$ General Hospital of Agios Nikolaos, Crete, Greece

${ }^{2}$ Venizeleio General Hospital of Heraklion, Crete, Greece

${ }^{3}$ University Hospital of Heraklion, Crete, Greece

*Corresponding author: loannis M Stavrakakis, MD, FEBOT, Locum Consultant Orthopaedics, General Hospital of Agios Nikolaos, 4 Knossou, Agios Nikolaos, PC 72100, Crete, Greece, Tel: 00302841343419

\begin{abstract}
Fractures of the sustentaculum tali and body of the talus are rare high energy injuries. Thorough clinical examination as well as proper imaging studies are necessary in order not to miss this kind of fractures. Severity of the injury, amount of displacement, soft tissue compromise, accuracy of reduction as well as stability of fixation are the main factors affecting the outcome. This study presents a case of a 19-year-old patient, who sustained a sustentaculum tali fracture with a concomitant lateral talar body fracture after a motor vehicle accident. Open reduction and internal fixation were performed. Sustentaculum tali fracture was approached through a plan between the posterior tibialis and the flexor digitorum longus tendon. Fibular door osteotomy was required in order to approach and fix the lateral talar body fracture. The surgical technique is described in details. Postoperative protocol and six months post-surgery outcome are demonstrated as well.
\end{abstract}

\section{Introduction}

Sustentaculum tali and talar body fractures are rare injuries resulting from high energy trauma in young patients $[1,2]$. Concomitant fractures of these structures on the same foot are even rarer. Failing to evaluate and treat these injuries properly leads to a significant disability $[1,3]$. This study highlights the importance of diagnosis and treatment of this kind of injury, through a case description. To the best of our knowledge this case is one of the few described in the literature.

We describe the surgical technique, which we performed, as well as the postoperative protocol followed for this case. Six months outcome is also reported, using the American Orthopaedic Foot and Ankle Society Score (AOFAS) [4].

\section{Case Report}

A 19-year-old patient presented to the emergency department of our institution after sustaining a left foot injury due to a motor vehicle injury. The patient was unable to bear weight, because of severe pain around the left ankle joint. On examination, significant swelling around the ankle and midfoot was noticed. Ankle range of motion was significantly reduced and palpable tenderness was located over the medial side just distal to the medial malleolus and over the lateral side just distal and anterior to the lateral malleolus. Hindfoot varus malalignment was also noticed on inspection. Patient reported a past medical history of an old sprain on the same ankle, which he was asymptomatic for.

Anteroposterior, mortise and true lateral views of the left foot and ankle radiographs were obtained (Figure 1 and Figure 2). A displaced lateral talar body fracture and subtalar joint disruption, as well as a tilt of the tibiotalar joint, were revealed from the plain radiographs. Osteochondral defect on the medial side of the dome of the talus, due to an old ankle injury, sustained few years ago, was also visible. CT scan of the left foot confirmed a displaced lateral talar body fracture along with a displaced sustentaculum tali fracture (Figure 3, Figure 4 and Figure 5). 


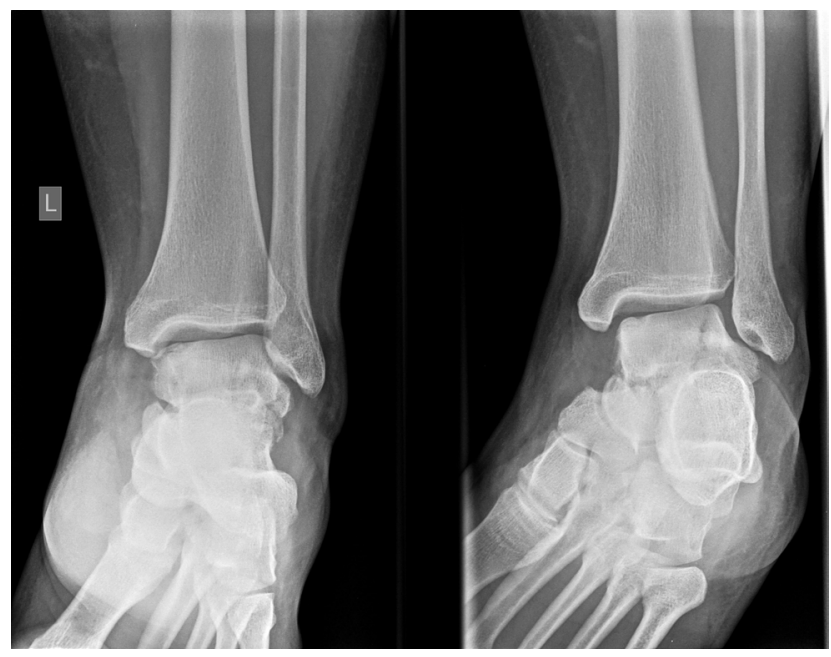

Figure 1: Ankle AP and mortise view. Lateral talar body fracture is noticed.

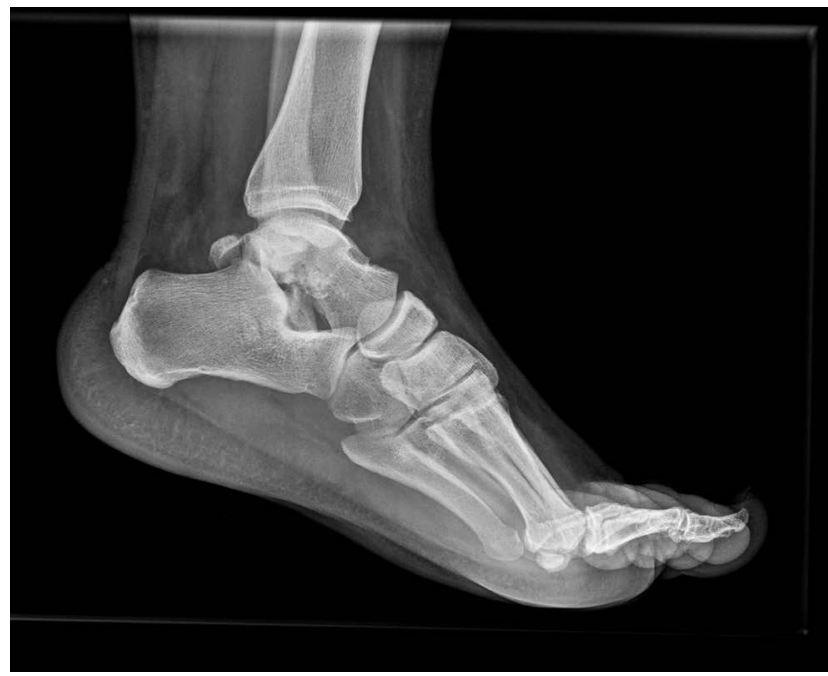

Figure 2: Foot and Ankle true lateral view. Sustentaculum tali fracture and subtalar disruption due to talar body fracture is identified.

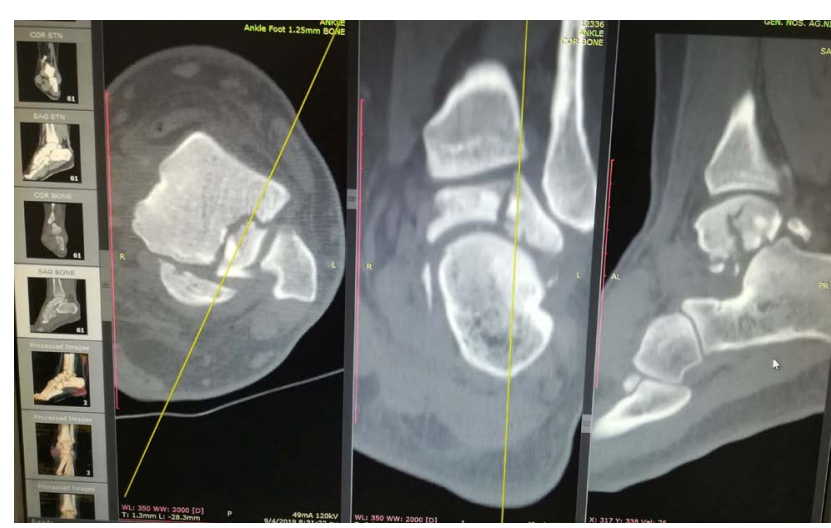

Figure 3: Ankle CT scan showing lateral talar body fracture with subtalar subluxation.

Due to the foot excessive swelling, a back slab was applied, immobilizing temporarily the ankle joint. Leg elevation, ice, and no weight bearing were instructed to the patient, who was admitted in the orthopaedic department for surgery 12 days later, as soon as the

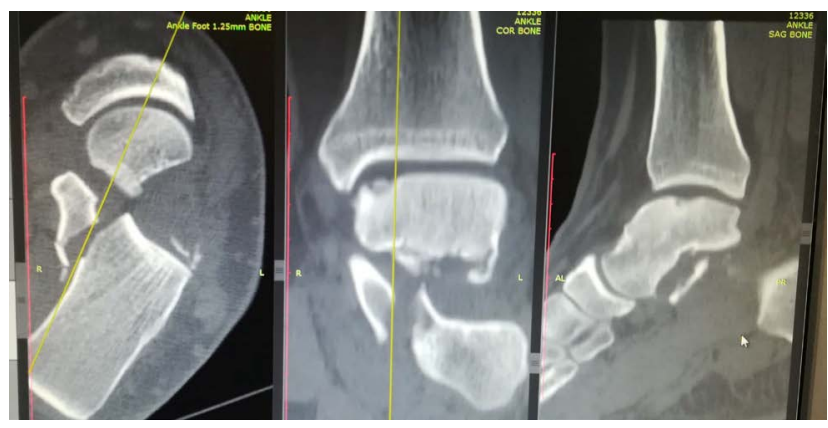

Figure 4: Ankle CT scan showing sustentaculum tali fracture with gross displacement.

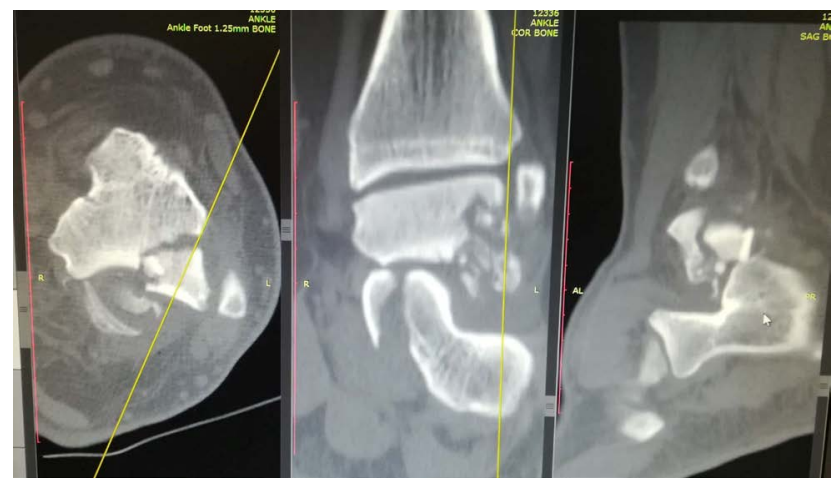

Figure 5: Ankle CT scan showing lateral talar process fracture with comminution.

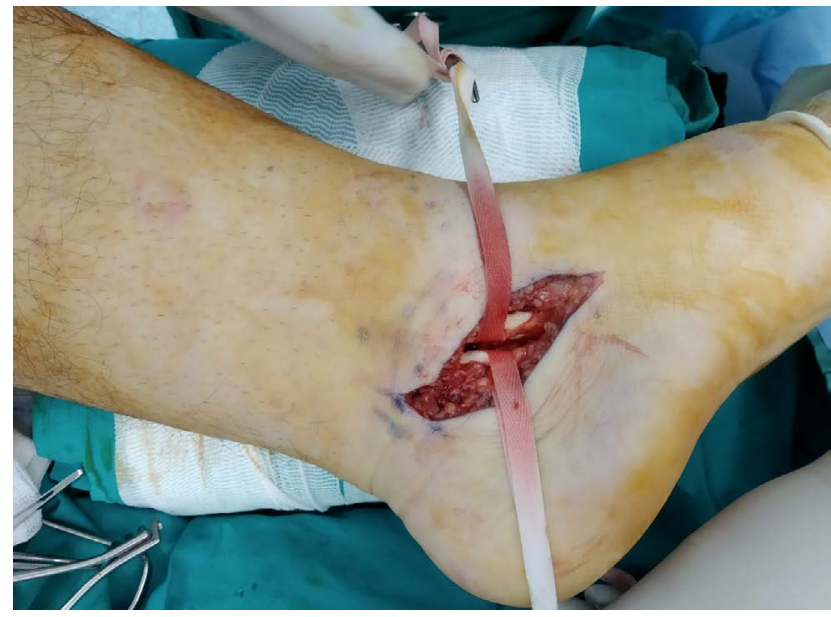

Figure 6: Intraoperative view. Surgical plan between tibialis posterior and flexor digitorum longus tendon.

swelling was subsided.

The patient was applied supine on the surgical table and a thigh tourniquet was applied. A medial incision 2 $\mathrm{cm}$ distal to the tip of the medial malleolus was performed, extending to the tuberosity of the navicular bone. Tibialis posterior and flexor digitorum longus were identified and protected (Figure 6). The plan between those two tendons was used to approach the sustentaculum (Figure 7). Care was taken not to violate the neurovascular structures, which they normally course posteriorly and inferiorly to the flexor digitorum longus. The fracture of the sustentaculum tali was identified, reduced under direct visual- 


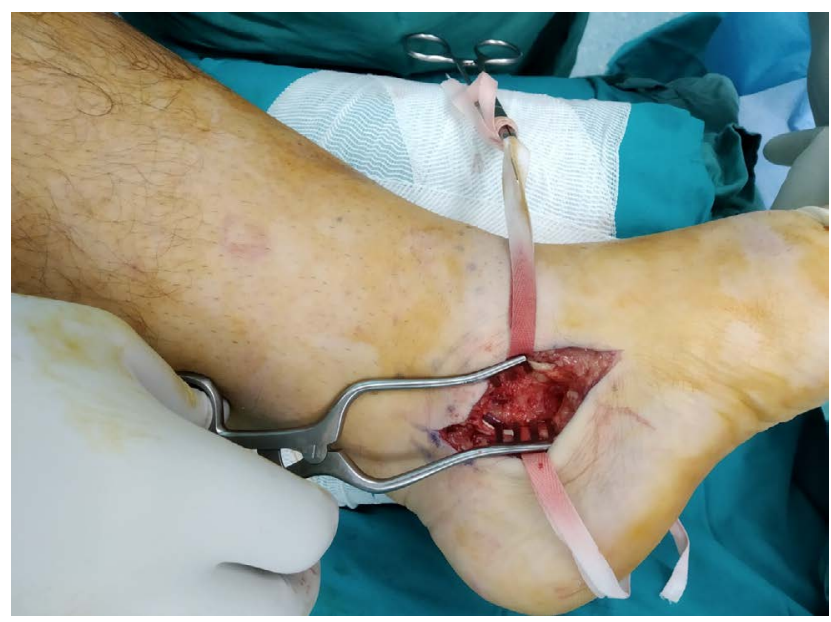

Figure 7: Intraoperative view. Sustentaculum tali fracture exposure.

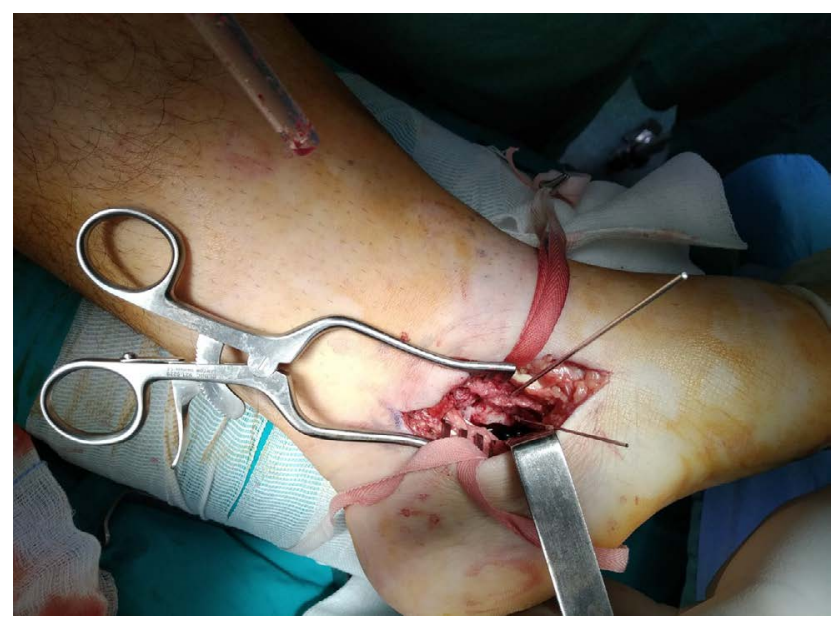

Figure 8: Intraoperative view. Fracture reduction and provisional stabilization with $\mathrm{KW}$.

ization of the medial calcaneal cortex and stabilized provisionally with Kirschner Wires (KW), under fluoroscopic control (Figure 8). Two spongiosa, partially threaded, cannulated $4.0 \mathrm{~mm}$ screws were used to fix the fracture. A buttress plate was also applied for stronger stabilization. An anterolateral ankle incision was performed starting approximately $4 \mathrm{~cm}$ above the level of the ankle joint extending distally, just proximally to the base of the $4^{\text {th }}$ metatarsal. As it was decided preoperatively, a distal fibular door osteotomy was performed in order to approach the fracture of the lateral talar body. Anterior inferior tibiofibular ligament (AITFL) and the anterior talofibular ligament (ATFL) were sharply dissected. Distal fibula was transversely osteotomized $2 \mathrm{~cm}$ above the ankle joint. The distal part of the osteotomy was externally rotated revealing the area of the fracture. Fracture of the lateral talar body was fixed with two partially threaded cannulated compression headless $3.0 \mathrm{~mm}$ screws. Fluoroscopic imaging confirmed the correct reduction of the fracture and position of the screws. Distal fibula was fixed with one third tubular plate. AITFL and ATFL were repaired with a $2 / 0$ absorbable suture. Postoperative radiographs were shown (Fig-

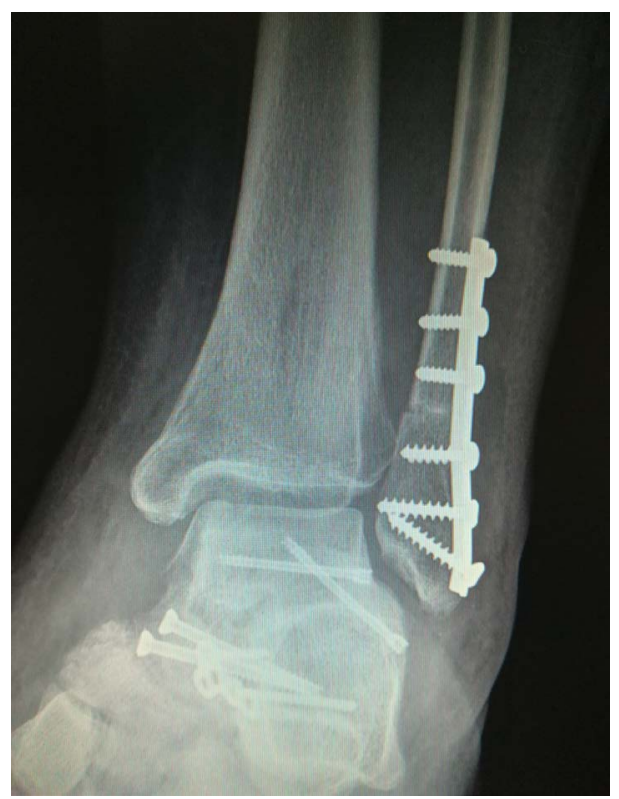

Figure 9: Post-operation ankle AP view.

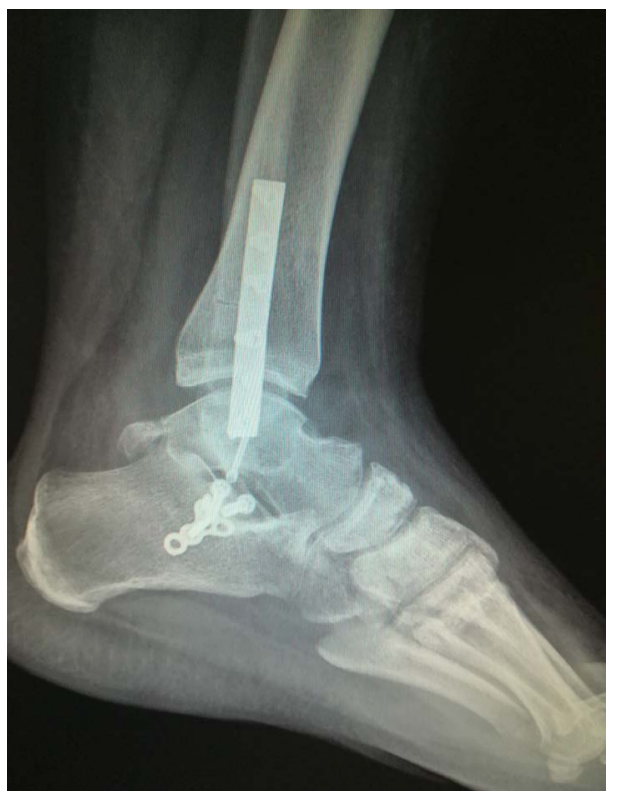

Figure 10: Post-operation foot and ankle true lateral view.

ure 9, Figure 10 and Figure 11).

A back slab of the ankle joint was applied and no weight bearing was instructed to the patient for 6 weeks. By the time the back slab was removed, the patient was encouraged to perform ankle range of motion, but no weight bearing was continued for another 6 weeks. Full weight bearing after 12 weeks post-surgery was advised to the patient and physiotherapy was initiated. After six months post-surgery the patient was pain free with normal ankle range of motion, moderate swelling, and scoring an AOFAS score of $90 \%$. Patient has been informed though that, despite the good radiographic result, complications, such as chronic pain due to avascular necrosis or joint posttraumatic degenerative changes are likely to occur in the future. 


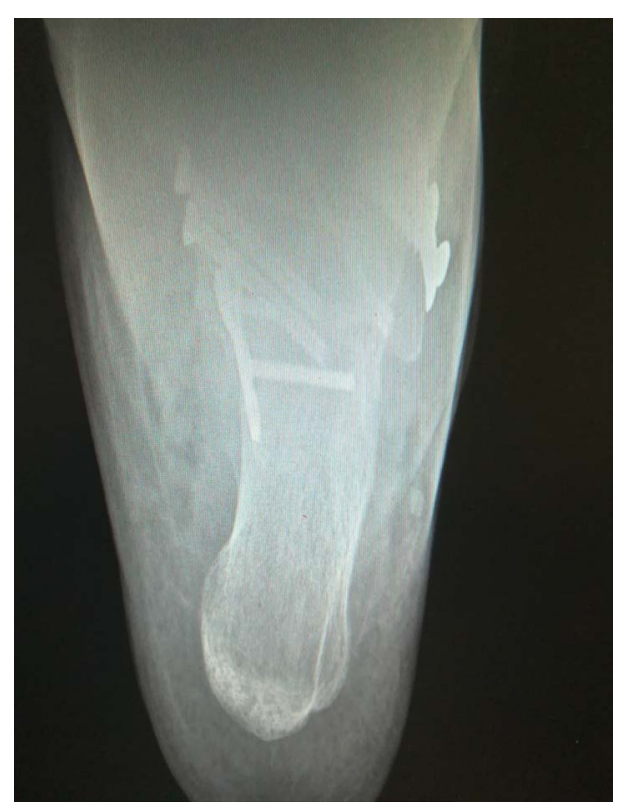

Figure 11: Post-operation Harris view, where acceptable reduction of the sustentaculum tali fracture is noticed.

\section{Discussion}

Fractures of the sustentaculum tali are rare and they are often associated with other foot injuries $[1,5]$. In this particular case a sustentaculum tali fracture along with a concomitant lateral talar body fracture occurred. Fractures of the sustentaculum tali are considered to be intraarticular, indicating anatomical reduction and fixation in case of displacement, as an optimal method of treatment [5]. Leaving a displaced sustentaculum tali fracture untreated may lead to hindfoot varus malalignment, chronic pain due to subtalar joint arthritis or flexor hallucis longus tendon impingement and tarsal tunnel syndrome [1,5]. Medial foot approach has been described as the best way of reducing and fixing these fractures. A longitudinal incision approximately $5 \mathrm{~cm}$ long, $2 \mathrm{~cm}$ distal to the tip of the medial malleolus aiming to the tuberosity of the navicular bone is recommended [5]. The tibialis posterior is identified and it is retracted dorsally, whereas the flexor digitorum longus and the flexor hallucis longus are mobilized and retracted distally, protecting the neurovascular bundle which runs between them. Periosteal striping will reveal the fracture which can then be reduced anatomically and fixed with either interfragmentary screws or/and plate $[1,5]$. Intraoperative Harris fluoroscopy view is required in order to evaluate the accuracy of lag screws' correct placement [6]. Using lag screws with a buttress plate makes fixation biomechanically more stable, allowing probably for earlier weight bearing.

Fractures of the body of the talus are rare, accounting for $0.62 \%$ of all fractures and $7 \%$ to $38 \%$ of all talar fractures [7]. Preoperative CT scan is necessary for essential planning prior to surgery $[7,8]$. Timing of surgery depends on the type of fracture. Provided that there is no gross fracture dislocation, surgery can be postponed until the swelling has been significantly subsided and the skin wrinkles. In case of a severe fracture dislocation, initial closed reduction through small incisions, if needed, on an urgent basis, is desirable, protecting the surrounding soft tissue $[9,10]$. There is though literature supporting delayed definitive treatment even for displaced closed fractures of the talus. Early open reduction and internal fixation is required for open displaced talar fractures dislocation [9]. Fracture pattern dictates the appropriate approach as well as malleolar osteotomy needed for fixation $[10,11]$. There are several distal fibular osteotomies described in the literature, especially for osteochondral lesions of the talus, such as syndesmosis preserving fibular osteotomy [12], or fibular window osteotomy [13]. Fibular door osteotomy, which was used in the presented case, is indicated when the talar fracture involves the lateral process and extends through the lateral body up to the talar plafond [2]. This technique usually requires AITFL and ATFL disruption, which can be repaired later [7].

Postoperatively a non weight bearing ankle cast is applied for 6 weeks, followed by another 6 weeks of non weight bearing ankle range of motion. Full weight bearing is allowed 12 weeks post-surgery [7].

It is well reported in the literature that fractures of the body of the talus are associated with several early and late complications. Wound dehiscence occurs frequently, especially if the swelling has not been subsided before surgery. Superficial skin infection occurs in $6.2 \%$ and deep infections in $3.1 \%$. Tibiotalar or subtalar joint arthritis ranges from $27 \%$ to $100 \%$. Avascular necrosis (AVN) ranges from $27 \%$ to $50 \%$ [2]. A radiolucent line on the ankle AP radiograph, visible 4 to 8 weeks post-surgery, is the so called Hawkins sign, which is an evidence of talus revascularization [8]. The higher the severity and displacement of the fracture dislocation, the higher the incidence of post-traumatic arthritis and $\operatorname{AVN}[2,10]$. Our case resulted in a good outcome, but a longer follow up is needed for safe conclusions.

\section{Conclusion}

This article presents a rare case of a concomitant ipsilateral displaced sustentaculum tali fracture and lateral talar body fracture, treated with open reduction and internal fixation, leading to a good outcome.

Authors declare that there is no conflict of interest.

\section{References}

1. Dürr C, Zwipp H, Rammelt S (2013) Fractures of the sustentaculum tali. Oper Orthop Traumatol 25: 569-578.

2. Sundararajan $S R$, Badurudeen AA, Ramakanth R, Rajasekaran S (2018) Management of talar body fractures. Indian J Orthop 52: 258-268.

3. Gatha M, Pedersen B, Buckley R (2008) Fractures of the sustentaculum tali of the calcaneus: A case report. Foot Ankle Int 29: 237-240. 
4. Cook JJ, Cook EA, Rosenblum BI, Landsman AS, Roukis TS (2011) Validation of the American College of foot and ankle surgeons scoring scales. J Foot Ankle Surg 50: 420429.

5. Della Rocca GJ, Nork SE, Barei DP, Taitsman LA, Benirschke SK (2009) Fractures of the sustentaculum tali: Injury characteristics and surgical technique for reduction. Foot Ankle Int 30: 1037-1041.

6. Gitajn IL, Toussaint RJ, Kwon JY (2013) Assessing accuracy of sustentaculum screw placement during calcaneal fixation. Foot Ankle Int 34: 282-286.

7. Early JS (2004) Management of fractures of the talus: Body and head regions. Foot Ankle Clin 9: 709-722.

8. Kilic A, Kabukcuoglu Y, Sokucu S (2009) The treatment of talar body fractures with compression screws: A case series. Cases J 2: 7953.
9. Rammelt S, Zwipp H (2009) Talar neck and body fractures. Injury 40: 120-135.

10. Vallier HA (2015) Fractures of the Talus: State of the art. J Orthop Trauma 29: 385-392.

11. Muir D, Saltzman CL, Tochigi $Y$, Amendola N (2006) Talar dome access for osteochondral lesions. Am J Sports Med 34: $1457-1463$.

12. Vaghela KR, Clement $H$, Parker $L$ (2016) Syndesmosis preserving osteotomy of the fibula for access to the lateral talar dome. Foot Ankle Surg 22: 210-213.

13. Scott D Allen, Christopher W DiGiovanni (2003) Distal fibular window osteotomy for exposure of lateral talar osteochondral lesion. Techniques in Foot and Ankle Surgery 2: 129-134. 